\title{
Early corticosteroids are associated with lower mortality in critically ill patients with COVID-19: a cohort study
}

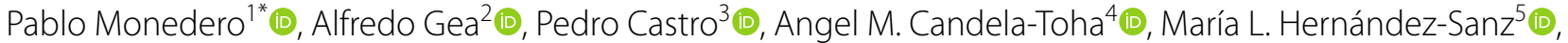 \\ Egoitz Arruti ${ }^{6}$, Jesús Villar $7,8,9$ and Carlos Ferrando $7,10 \mathbb{D}$ on behalf of for the COVID-19 Spanish ICU Network
}

\begin{abstract}
Background: Critically ill patients with coronavirus disease 19 (COVID-19) have a high fatality rate likely due to a dysregulated immune response. Corticosteroids could attenuate this inappropriate response, although there are still some concerns regarding its use, timing, and dose.

Methods: This is a nationwide, prospective, multicenter, observational, cohort study in critically ill adult patients with COVID-19 admitted into Intensive Care Units (ICU) in Spain from 12th March to 29th June 2020. Using a multivariable Cox model with inverse probability weighting, we compared relevant outcomes between patients treated with early corticosteroids (before or within the first $48 \mathrm{~h}$ of ICU admission) with those who did not receive early corticosteroids (delayed group) or any corticosteroids at all (never group). Primary endpoint was ICU mortality. Secondary endpoints included 7-day mortality, ventilator-free days, and complications.
\end{abstract}

Results: A total of 691 patients out of 882 (78.3\%) received corticosteroid during their hospital stay. Patients treated with early-corticosteroids $(n=485)$ had lower ICU mortality (30.3\% vs. never 36.6\% and delayed 44.2\%) and lower 7-day mortality (7.2\% vs. never 15.2\%) compared to non-early treated patients. They also had higher number of ventilator-free days, less length of ICU stay, and less secondary infections than delayed treated patients. There were no differences in medical complications between groups. Of note, early use of moderate-to-high doses was associated with better outcomes than low dose regimens.

Conclusion: Early use of corticosteroids in critically ill patients with COVID-19 is associated with lower mortality than no or delayed use, and fewer complications than delayed use.

Keywords: COVID-19, Intensive Care Unit, Corticosteroids, Critically ill patient, Cohort study, Outcomes, Ventilatorfree days, Mortality

\section{Background}

Coronavirus disease 2019 (COVID-19), caused by the severe acute respiratory syndrome coronavirus 2 (SARS-CoV-2), was first recognized in Wuhan, China,

\footnotetext{
*Correspondence: pmonedero@unav.es

${ }^{1}$ Department of Anaesthesiology and Intensive Care, Clínica Universidad de Navarra, Pio XII, 36, 31008 Pamplona, Spain

Full list of author information is available at the end of the article Investigators of the COVID-19 Spanish ICU Network are listed in the Acknowledgments section ofthe main manuscript
}

in December 2019 [1]. The antiviral immune response is crucial to eliminate the invading virus. However, an inappropriate response may cause a systemic hyperinflammatory state, producing complications such as acute respiratory distress syndrome (ARDS) and multisystem organ failure [2]. Early treatment of this hyperinflammation may be important for reducing mortality in COVID19 patients.

Corticosteroids are used to treat several hyperinflammatory syndromes [3]. Early after the outbreak, the original author(s) and the source, provide a link to the Creative Commons licence, and indicate if changes were made. The images or other third party material in this article are included in the article's Creative Commons licence, unless indicated otherwise in a credit line to the material. If material is not included in the article's Creative Commons licence and your intended use is not permitted by statutory regulation or exceeds the permitted use, you will need to obtain permission directly from the copyright holder. To view a copy of this licence, visit http://creativecommons.org/licenses/by/4.0/. The Creative Commons Public Domain Dedication waiver (http://creativeco mmons.org/publicdomain/zero/1.0/) applies to the data made available in this article, unless otherwise stated in a credit line to the data. 
World Health Organization (WHO) recommended against the routine use of systemic corticosteroids for treating COVID-19 patients, due to their known side effects and a potential slowing of viral clearance [1]. The RECOVERY trial [4] and six smaller randomized clinical trials have shown improved outcomes in severe COVID-19 patients treated with corticosteroids [4-6]. Consequently, the US National Institutes of Health (NIH) recommends the use of dexamethasone to treat COVID-19 patients requiring supplemental oxygen [7], and the WHO stated a strong recommendation for systemic corticosteroid therapy in patients with severe and critical COVID-19, and a conditional recommendation not to use corticosteroid therapy in patients with nonsevere COVID-19 [8]. However, the RECOVERY trial has methodological flaws such as the absence of stratification and incomplete information on multiple factors associated with mortality, which may have caused an imbalance between the control and the corticosteroids treated groups [9]. The WHO meta-analysis [5] also has limitations, with 3 incomplete trials stopped prematurely, and excessive weight of the RECOVERY trial, precluding definitive conclusions [9].

Spain is one of the European countries most affected by the COVID-19 pandemic with a broad experience in the use of corticosteroids in Intensive Care Units (ICU) for patients with ARDS [10]. In a large COVID-19 registry of patients admitted into a network of ICUs, we examined whether early use of corticosteroids decreases all-cause mortality and improves clinically relevant outcomes.

\section{Methods}

\section{Study design}

This is a multicentre, observational study with retrospective analysis of prospectively collected data in consecutive critically ill COVID-19 patients admitted from 12th March to 29th June 2020 into a network of ICUs in 36 hospitals from Spain and Andorra. The study was approved by a referral Ethics Committee (Ethics Committee of Euskadi, Spain) and by all participating hospitals.

\section{Data source and study population}

Following a standardized protocol, site investigators collected data from electronic medical records. We recorded pre-admission and daily data from ICU admission to ICU discharge. Before data analysis, two independent investigators and a statistician screened the database for errors against standardized ranges and contacted site investigators with queries.

All consecutive COVID-19 patients admitted to participating ICUs were considered for study entry if they had: age $\geq 18$ years and confirmed SARS-CoV- 2 infection from a respiratory tract sample using RT-PCR assay. Exclusion criteria were non-confirmed SARS-CoV-2 infection, patients with no data at baseline, patients with do-not-resuscitate orders, and patients who did not meet the outcomes of death or ICU discharge by 29th June 2020.

\section{Variables, exposures, and endpoints}

We recorded data on demographics and comorbidities according to established definitions (See Additional file 1), laboratory findings, vital signs, severity scores at ICU admission, supportive therapies, and relevant outcomes reported by 29th June 2020. We collected preICU-admission and full data set on the first day of ICU (baseline), and the "worst" values during ICU stay (maximum or minimum, depending on the parameter).

For this study, we established a post hoc cut-off at $48 \mathrm{~h}$ after ICU admission, based on the comparison of survivors vs. non-survivors, and because it was a reasonable time for a clinician to decide whether therapy with corticosteroids should be initiated based on the initial ICU evolution and the laboratory results (inflammation and organ failure). So, we classified patients in three groups: (i) patients receiving corticosteroids within the first $48 \mathrm{~h}$ (early group); (ii) those receiving corticosteroids after $48 \mathrm{~h}$ of ICU admission (delayed group); and (iii) those who never received corticosteroids (never group). The union of delayed and never groups is analysed as nonearly group. Other exploratory exposures included the administration of corticosteroids at any time during hospital stay (ever-treated group =early plus delayed use) and no administration at all (never-treated group). We also examined patients receiving low dose of corticosteroids (defined as methylprednisolone $<1 \mathrm{mg} / \mathrm{kg} / \mathrm{d}$ or dexamethasone $<0.12 \mathrm{mg} / \mathrm{kg} / \mathrm{d}$ or prednisone $<0.5 \mathrm{mg} / \mathrm{kg} / \mathrm{d}$ ) or receiving moderate-to-high doses (any dose higher than low dose).

The primary endpoint was ICU mortality. We excluded from the analysis patients who died or were discharged within the first $48 \mathrm{~h}$. Secondary endpoints were medical and infectious complications, ventilator-free days, ICU length of stay (LOS), and 7-day mortality.

\section{Statistical analysis}

We aimed to enrol as many patients as possible, with no pre-defined sample size.

We report the values of variables as percentages, mean and standard deviation (SD), or median and interquartile range (IQR), as appropriate. To compare variables among groups, we used Student $t$ test or Mann-Whitney test and one-way ANOVA or Kruskal-Wallis test for numerical variables, and Chi-squared test or Fisher exact test for categorical variables. 
To assess the relationship between corticosteroids treatment and endpoints, time-to-event curves were plotted using the Kaplan-Meier method and analysed with Cox regression analysis. For the Kaplan-Meier curves, patients with complementary outcomes were right-censored at the longest recorded LOS. We used inverse probability of treatment weighting (IPW) for baseline differences between treatment groups. We fitted logistic models using the following baseline variables: age, gender, comorbidities (diabetes mellitus and arterial hypertension), APACHE II and SOFA scores, and $\mathrm{PaO}_{2} /$ $\mathrm{FiO}_{2}$ at admission. Weights were calculated following the methodology described elsewhere [11], and a pseudopopulation (adjusted sample) was built subsequently. The 95\% confidence intervals (CI) were the 2.5 th and 97.5th percentiles of the distribution obtained from a nonparametric bootstrap with 1,000 samples. To test the robustness of results, we rerun the primary analysis under several assumptions and scenarios (See Additional file 1).
Furthermore, to assess the relationship between survival and onset of corticosteroids treatment, we fitted a logistic regression where mortality was the dependent variable, and day of starting corticosteroids treatment was the independent variable included as continuous in a linear model, and a restricted cubic splines model to account for potential nonlinear relationships between corticosteroids onset and mortality.

Missing data were not imputed. Analyses were performed in a complete case analysis basis. All tests were two-sided, and a p value $<0.05$ was considered statistically significant. Analyses were performed using STATA version 16.

\section{Results}

From 1,102 consecutive patients with COVID-19, we analysed 882 patients (Fig. 1). Baseline characteristics are reported in Table 1. Acute hypoxemic respiratory failure was the main reason for ICU admission.

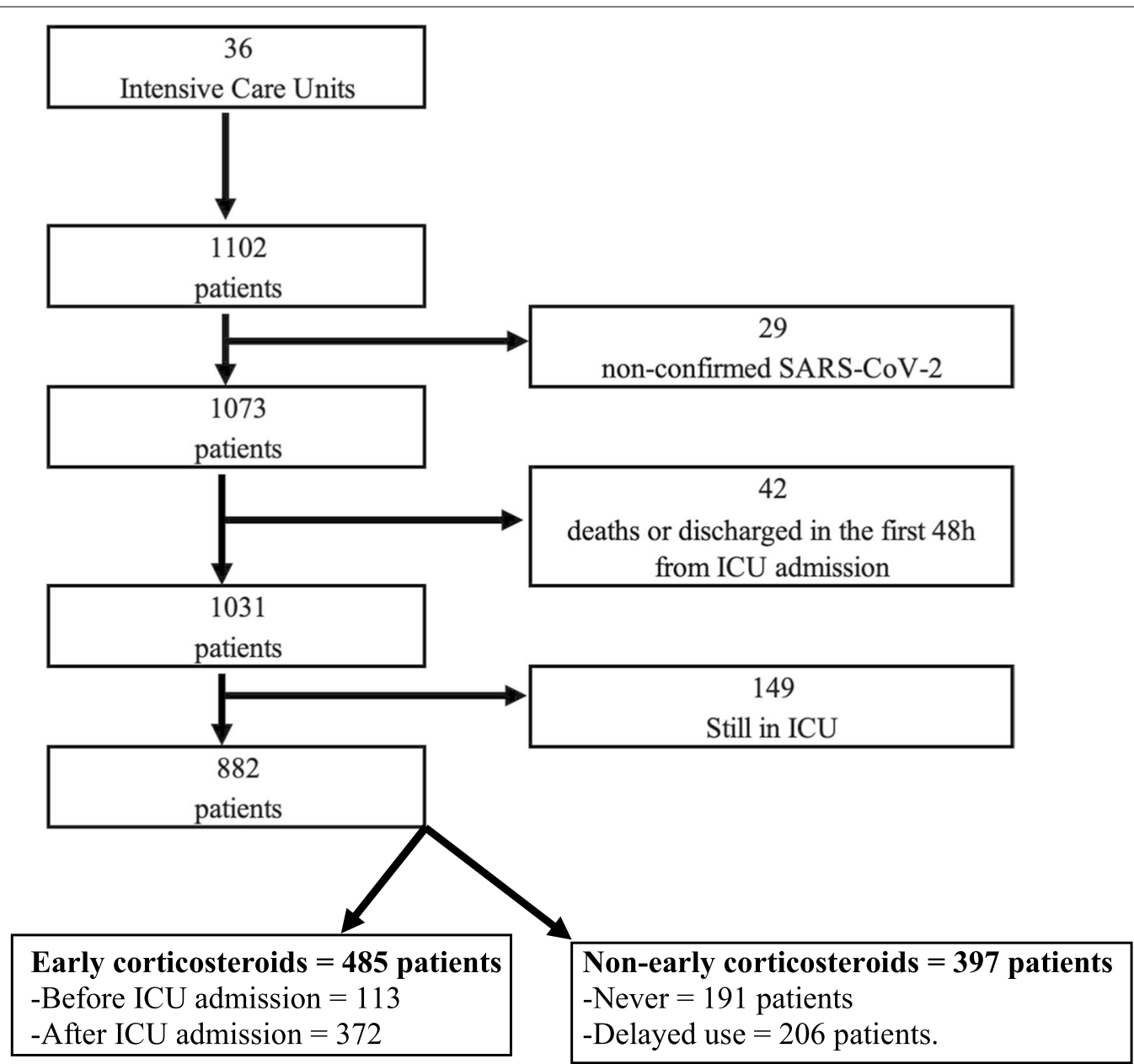

Fig. 1 Flow chart of study participants. ICU=Intensive Care Unit 
Table 1 Baseline characteristics of patients according to the use of corticosteroids. Delayed $=$ after $48 \mathrm{~h}$ of ICU admission

\begin{tabular}{|c|c|c|c|c|c|}
\hline & Whole cohort & Never & Early & Delayed & $p$ value \\
\hline \multicolumn{6}{|c|}{ Patients demographics and comorbidities } \\
\hline N (\%) & 882 & $191(21.66 \%)$ & 485 (54.99\%) & $206(23.36 \%)$ & \\
\hline Age, years & $62.3(11.4)$ & $60.1(13.9)^{\mathrm{a}}$ & $62.9(10.6)$ & $63.0(10.8)$ & 0.06 \\
\hline Female (\%) & $291(33.1 \%)$ & $66 / 191(34.5 \%)$ & $153 / 485(31.5 \%)$ & $72 / 204(35.2 \%)$ & 0.56 \\
\hline Body mass index $\left(\mathrm{kg} / \mathrm{m}^{2}\right)$ & $29.2(5.3) / 550$ & $28.71(5.4) / 114$ & $29.2(5.3) / 292$ & $29.5(5.3) / 144$ & 0.39 \\
\hline Arterial hypertension & $420 / 882(47.6 \%)$ & 79/191 (41.4\%) & $240 / 485(49.4 \%)$ & $101 / 206(49.0 \%)$ & 0.14 \\
\hline Diabetes mellitus & 201/882 (22.7\%) & 43/191 (22.5\%) & $111 / 485(22.9 \%)$ & $47 / 206(22.8 \%)$ & 1.00 \\
\hline Chronic heart failure & $13 / 882(1.4 \%)$ & $1 / 191(0.5 \%)$ & $7 / 485(1.4 \%)$ & $5 / 206(2.4 \%)$ & 0.26 \\
\hline Chronic renal failure & $52 / 882(5.9 \%)$ & 11/191 (5.7\%) & 28/485 (5.7\%) & $13 / 206(6.3 \%)$ & 0.96 \\
\hline Asthma & $24 / 882(2.7 \%)$ & $5 / 191(2.6 \%)$ & $16 / 485(3.3 \%)$ & $3 / 206(1.4 \%)$ & 0.42 \\
\hline COPD & $40 / 882(4.5 \%)$ & $5 / 191(2.6 \%)$ & $24 / 485(4.9 \%)$ & $11 / 206(5.3 \%)$ & 0.33 \\
\hline Obese & 299/792 (37.7\%) & $66 / 170(38.8 \%)$ & 158/435 (36.3\%) & 75/187 (40.1\%) & 0.63 \\
\hline Dyslipidaemia & 132/882 (14.9\%) & 21/191 (11.0\%) & 76/485 (15.6\%) & $35 / 206(17.0 \%)$ & 0.18 \\
\hline Cancer & 27/882 (3.1\%) & $3 / 191(1.6 \%)$ & $8 / 485$ (1.6\%) & 16/206 (7.8\%) & $<0.001$ \\
\hline \multicolumn{6}{|l|}{ Laboratory findings } \\
\hline Haematocrit (\%) & $39.2(5.7) / 602$ & $39.1(6.0) / 122$ & $39.3(5.6) / 313$ & $38.9(5.80) / 167$ & 0.62 \\
\hline Platelets, $1000 / \mathrm{mm}^{3}$ & $236(108) / 672$ & $232(106) / 130$ & $242(108) / 367$ & $222(108) / 175$ & 0.11 \\
\hline Leukocytes, $10^{3} / \mu \mathrm{L}$ & $9.1(5.8) / 663$ & $8.6(5.2) / 129$ & $9.4(6.1) / 360$ & $8.6(5.5) / 174$ & 0.10 \\
\hline Lymphocytes, $\mu \mathrm{L}$ & $0.82(0.72) / 658$ & $0.82(0.45) / 126$ & $0.84(0.87) / 361$ & $0.76(0.49) / 171$ & 0.16 \\
\hline $\mathrm{CRP}, \mathrm{mg} / \mathrm{dL}$ & $74.5(98.9) / 623$ & $85.4(110.9) / 119$ & $69.0(93.2) / 349$ & $78.5(101.2) / 155$ & 0.08 \\
\hline Lactate, mmol/L & $0.43(0.94) / 475$ & $0.39(0.53) / 99$ & $0.44(0.88) / 245$ & $0.46(1.26) / 131$ & 0.27 \\
\hline Ferritin, ng/mL & $1665(1658) / 285$ & 1744(2191)/38 & $1725(1669) / 179$ & $1459(1241) / 68$ & 0.52 \\
\hline D-Dimer, ng/mL & $2026(2339) / 522$ & $1586(1957) / 96^{\mathrm{a}}$ & $2184(2507) / 292$ & $1996(2177) / 134$ & 0.06 \\
\hline CRP/lymphocyte ratio & $131(234) / 617$ & $158(342) / 118$ & $110(168) / 344$ & $154(254) / 155$ & 0.08 \\
\hline IL-6, pg/mL & $253(486) / 87$ & $269(154) / 4$ & $289(543) / 62$ & $143(308) / 21$ & 0.08 \\
\hline $\mathrm{LDH}, \mathrm{U} / \mathrm{L}$ & $484(240) / 595$ & $453(268) / 114^{a}$ & $483(215) / 330$ & $508(264) / 151$ & 0.06 \\
\hline Procalcitonin, ng/mL & $1.36(5.1) / 454$ & $0.91(2.4) / 83$ & $1.15(4.0) / 260$ & $2.18(8.0) / 111$ & 0.44 \\
\hline Bilirubin, mg/dL & $0.82(1.16) / 567$ & $0.91(1.46) / 98$ & $0.76(0.61) / 322$ & $0.87(1.72) / 147$ & 0.89 \\
\hline $\mathrm{AST}, \mathrm{U} / \mathrm{L}$ & $58.3(86.6) / 650$ & $68.5(148.7) / 122$ & $57.2(60.1) / 359$ & $53.3(72.4) / 169$ & 0.45 \\
\hline Creatinine, mg/dL & $1.05(0.68) / 658$ & $1.07(0.76) / 124$ & $1.03(0.66) / 361$ & $1.07(0.66) / 173$ & 0.90 \\
\hline Urea, mg/dL & $46.5(28.0) / 462$ & $43.7(35.3) / 80$ & $46.6(27.4) / 265$ & $47.9(23.0) / 117$ & 0.05 \\
\hline NTProBNP, pg/mL & $1880(5166) / 96$ & $375(1278) / 64$ & $177(780) / 194$ & $89(451) / 83$ & 0.28 \\
\hline \multicolumn{6}{|l|}{ Vital signs and organ support } \\
\hline $\begin{array}{l}\text { Mechanical ventilation, } \\
\mathrm{n}(\%)\end{array}$ & 27/882 (3.1\%) & $2 / 191(1.0 \%)$ & $20 / 485(4.1 \%)$ & $5 / 206(2.4 \%)$ & 0.09 \\
\hline Vasopressor use, n (\%) & $377 / 882(42.7 \%)$ & 77/191 (40.3\%) & 214/485 (44.1\%) & $86 / 206(41.8 \%)$ & 0.64 \\
\hline $\begin{array}{l}\text { Renal replacement therapy, } \\
\text { n (\%) }\end{array}$ & $5 / 882(0.6 \%)$ & 2/191 (1.0\%) & $2 / 485(0.4 \%)$ & $1 / 206(0.5 \%)$ & 0.62 \\
\hline $\mathrm{PaO}_{2} / \mathrm{FiO}_{2}$ & $150(77) / 559$ & $153(78) / 107$ & $151(77) / 305$ & $146(76) / 147$ & 0.71 \\
\hline PEEP, $\mathrm{cmH} 2 \mathrm{O}$ & $13.8(2.6) / 257$ & $13.5(2.5) / 51$ & $14.0(2.5) / 136$ & $13.4(2.8) / 70$ & 0.15 \\
\hline $\mathrm{SpO}_{2}, \%$ & $88.1(9.2) / 641$ & $87.6(10.6) / 136$ & $88.4(7.8) / 342$ & $87.8(10.4) / 163$ & 0.84 \\
\hline Respiratory rate, bpm & $25.7(7.3) / 604$ & $26.6(8.7) / 126$ & $24.8(6.5) / 321$ & $26.6(7.3) / 157$ & 0.02 \\
\hline $\mathrm{PaCO} 2, \mathrm{mmHg}$ & $40.2(12.5) / 615$ & $40.4(13.7) / 128$ & $40.0(11.9) / 325$ & $40.5(12.7) / 162$ & 0.91 \\
\hline Temperature, ${ }^{\circ} \mathrm{C}$ & $36.9(1.1) / 654$ & $37.0(1.1) / 137$ & $36.7(1.0) / 345$ & $37.1(1.1) / 172$ & 0.001 \\
\hline $\begin{array}{l}\text { Mean arterial pressure, } \\
\mathrm{mmHg}\end{array}$ & $86.7(15.3) / 644$ & $86.9(14.8) / 134$ & $86.8(15.8) / 341$ & $86.3(14.7) / 169$ & 0.96 \\
\hline Heart rate, bpm & $85.2(18.8) / 658$ & $88.2(19.3) / 142$ & $84.0(18.9) / 346$ & $84.9(17.9) / 170$ & 0.18 \\
\hline \multicolumn{6}{|l|}{ Severity scores } \\
\hline Apache II & $13.51(6.35) / 600$ & $12.45(5.85) / 121$ & $13.77(6.67) / 348$ & $13.78(5.84) / 131$ & 0.17 \\
\hline CURB65 & $1.86(1.22) / 288$ & $1.67(1.26) / 60$ & $1.93(1.23) / 150$ & $1.90(1.16) / 78$ & 0.32 \\
\hline SOFA & $5.64(2.94) / 474$ & $5.85(3.18) / 86$ & $5.58(2.99) / 266$ & $5.60(2.65) / 122$ & 0.66 \\
\hline
\end{tabular}


Table 1 (continued)

\begin{abstract}
Statistically significant results are in italics
a Never significantly different from early and delayed; $p=0.02$

COPD: Chronic Obstructive Pulmonary Disease; CRP: C-reactive protein; LDH: lactate dehydrogenase; AST: aspartate aminotransferase; NTProBNP: N-terminal probrain natriuretic peptide; APACHE: Acute Physiology and Chronic Health Evaluation; CURB65: confusion, uremia, elevated respiratory rate, hypotension, and aged 65 years or older; SOFA: Sequential Organ Failure Assessment
\end{abstract}

Four-hundred and eighty-five patients (55.0\%) were treated with early corticosteroids (See Additional file 2: Table S1). Corticosteroid exposure did not differ according to age, sex, body-mass index, severity scores, and main comorbidities but small differences were present so that patients receiving early corticosteroids had lower temperature and respiratory rate, and the delayed group had more cancer patients (Table 1). During ICU stay, corticosteroid early treated patients developed less organ dysfunction, had less requirement for renal replacement therapy (RRT), and less systemic inflammation than delayed treated patients (Table 2).

\section{Primary outcome}

Overall ICU mortality was $34.9 \%(\mathrm{n}=308)$, significantly lower in the early corticosteroids group $(30.3 \%)$ than in non-early treated group (40.3\%) (HR 0.71, 95\% CI 0.57-0.89) (Table 3, Fig. 2). A sensitivity analysis showed less mortality reduction with corticosteroids in women, patients $<60$-year-old, with hypertension, cancer, or type 2 diabetes, $\mathrm{CRP}<10 \mathrm{mg} / \mathrm{dL}$, D-Dimer $>1500 \mathrm{ng} / \mathrm{mL}$, ICU admission $>8$ days after the onset of symptoms, corticosteroids within 7 days of symptom onset, $\mathrm{Pa} / \mathrm{FiO}_{2}>200$, or APACHE score > 14 at ICU admission (Additional file 3: Table S2). The reduction in mortality with corticosteroids was independent of treatment with tocilizumab, initiation of steroids before ICU admission, need of invasive mechanical ventilation (MV), lymphocytes count, duration of corticosteroids treatment, or the admitting hospital (Additional file 3: Table S2). This reduction in mortality was also observed when including the 149 patients not discharged from ICU or when including "missing" as a category in the IPW cohort.

We found a statistically significant linear association between the day of starting corticosteroids treatment and mortality (Additional file 4: Figure S1). However, patients who started corticosteroids before ICU admission showed a higher mortality rate (crude mortality $39.8 \%$ ) as compared to those who started corticosteroids after ICU admission (crude mortality 27.4\%). This difference disappeared in the adjusted model $(\mathrm{p}=0.40)$ (Additional file 1: Figure S2), pointing to a selection bias-those who failed to respond to corticosteroids were admitted to ICU-that precludes further conclusions about pre-ICU corticosteroids treatment. These results indicate that the earlier the better, after ICU admission.
ICU mortality was lower when using moderate-to-high doses of corticosteroids $(26.9 \%$ vs. $32.8 \%$ with low dose (HR 0.58, 95\% CI 0.45-0.75) (Additional file 1: Table S3S4, Additional file 1: Figure S3).

\section{Secondary outcomes}

Patients treated with early corticosteroids had shorter ICU LOS, more ventilatory-free days, lower rate of acute renal failure, less need of vasopressors, fewer infections and less inflammation and organ failure than delayed treated patients. There were no differences in the rate of medical complications between groups (Tables 2-3).

Regarding corticosteroids doses, early use of moderate-to-high doses, compared with low doses, was associated with a shorter ICU LOS, lower organ dysfunction, less requirement of $\mathrm{MV}$ or RRT, and no increase in medical or infectious complications (Additional file 1: Table S3-S4).

Patients who never received corticosteroids were a less severe population: younger, with fewer signs of organ damage and inflammation, and lower requirement for MV. Nonetheless, compared with early treated patients, they had higher ICU mortality $(36.6 \%$ vs. $30.3 \%$, HR $0.55,95 \%$ CI $0.35-0.93$ ) and higher early 7-days mortality (Table 3, Additional file 1: Figure S4). In contrast, compared with ever-treated patients, they had no difference in ICU survival, but lower LOS, less requirement for mechanical ventilation and vasopressors, better disease progression, and a lower number of infectious complications (Additional file 1: Tables S5-S6, Fig. 3).

\section{Discussion}

The major findings of our study are that early use of corticosteroids in critically ill patients with COVID-19 was associated with: (i) lower ICU mortality when compared to delayed or no use of corticosteroids; (ii) shorter ICU LOS; (iii) decreased organ dysfunction, and (iv) fewer days on MV, with no increase in medical or infectious complications. These findings remained statistically significant after adjusting for age, gender, comorbidities, severity, and $\mathrm{PaO}_{2} / \mathrm{FiO}_{2}$ at admission.

To study the influence of corticosteroids on mortality in our cohort, we set a cut-off point of $48 \mathrm{~h}$ after admission to ICU based on the comparison of survivors vs. nonsurvivors where we found that the onset time for using corticosteroids was clinically relevant. Furthermore, we 
Table 2 Evolution of organ failure, vital signs, and laboratory findings during ICU stay according to the use of corticosteroids. Early $=$ in the first $\mathbf{4 8} \mathrm{h}$ of ICU admission

\begin{tabular}{|c|c|c|c|c|c|}
\hline & Whole cohort & Never & Early & Delayed & $p$ value \\
\hline n (\%) & 882 & $191(21.7 \%)$ & $485(55.0 \%)$ & $206(23.3 \%)$ & \\
\hline Mechanical ventilation, $\mathrm{n}(\%)$ & $722 / 882(81.9 \%)$ & $139 / 191(72.8 \%)^{\mathrm{a}}$ & $395 / 485(81.4 \%)$ & 188/206 (91.3\%) & $<0.001$ \\
\hline Vasopressor use, n (\%) & $639 / 882(72.4 \%)$ & 126/191 (66.0\%) & $343 / 485(70.7 \%)$ & $170 / 206(82.5 \%)^{c}$ & $<0.001$ \\
\hline Renal replacement therapy, n (\%) & $96 / 867(11.1 \%)$ & $14 / 183(7.6 \%)$ & $40 / 479(8.3 \%)$ & $42 / 205(20.5 \%)^{c}$ & $<0.001$ \\
\hline SOFA maximum & $8.6(3.7) / 729$ & $8.2(3.5) / 132$ & $8.2(3.5) / 426$ & $9.7(4.0) / 171^{c}$ & $<0.001$ \\
\hline \multicolumn{6}{|l|}{ Vital Signs } \\
\hline Temperature maximum, ${ }^{\circ} \mathrm{C}$ & $37.9(1.0) / 869$ & $37.9(1.0) / 183$ & $37.7(1.0) / 481^{b}$ & $38.2(1.0) / 205$ & $<0.001$ \\
\hline Mean arterial pressure minimum, $\mathrm{mmHg}$ & $68.8(13.0) / 868$ & $70.7(13.4) / 182$ & $69.1(13.0) / 480$ & $66.4(12.3) / 206^{c}$ & 0.001 \\
\hline Heart rate maximum, bpm & $105.6(20.9) / 868$ & $103.9(21.7) / 182$ & $104.6(20.9) / 480$ & $109.5(19.9) / 206^{c}$ & 0.006 \\
\hline $\mathrm{SpO}_{2}$, minimum, $\%$ & $78.6(16.2) / 869$ & $80.3(16.2) / 184$ & $79.4(15.1) / 480$ & $75.3(18.2) / 205^{c}$ & $<0.001$ \\
\hline Respiratory rate, maximum, bpm & $30(7.0) / 859$ & $31(7.7) / 177$ & $30(6.6) / 477$ & $32(7.2) / 205^{c}$ & 0.005 \\
\hline Respiratory rate, minimum, bpm & $17(4.4) / 859$ & $19(5.0) / 177^{a}$ & $17(4.4) / 477$ & $16(3.8) / 205$ & $<0.001$ \\
\hline \multicolumn{6}{|l|}{ Arterial blood gas } \\
\hline $\mathrm{PaO}_{2} / \mathrm{FiO}_{2}$ minimum & $98.3(47.9) / 833$ & $107.6(56.9) / 167^{\mathrm{a}}$ & $96.6(43.5) / 466$ & $94.3(48.5) / 200$ & 0.03 \\
\hline $\mathrm{PaCO}_{2}$ maximum, $\mathrm{mmHg}$ & $61.8(19.3) / 855$ & $65.0(21.2) / 178$ & $58.9(18.0) / 473^{b}$ & $66.4(19.3) / 204$ & $<0.001$ \\
\hline \multicolumn{6}{|l|}{ Laboratory findings } \\
\hline Ferritin maximum, ng/mL & $2305(2257) / 667$ & $1771(1863) / 104^{\mathrm{a}}$ & $2314(2251) / 389$ & $2604(2432) / 174$ & 0.003 \\
\hline D-Dimer maximum, $\mathrm{ng} / \mathrm{mL}$ & $5124(2983) / 809$ & $4124(2823) / 158^{\mathrm{a}}$ & $5176(3008) / 452$ & $5799(2848) / 199$ & $<0.001$ \\
\hline CRP maximum, mg/dL & $121(138) / 845$ & $124(140) / 172$ & $111(128) / 472$ & $142(154) / 201^{c}$ & 0.005 \\
\hline Lymphocytes minimum, $\mathrm{mL}$ & $0.45(0.34) / 848$ & $0.60(0.41) / 175^{\mathrm{a}}$ & $0.42(0.31) / 471$ & $0.39(0.29) / 202$ & $<0.001$ \\
\hline CRP/lymphocyte ratio maximum & $273(447) / 843$ & $237(382) / 171$ & $245(389) / 471$ & $368(590) / 201^{c}$ & 0.004 \\
\hline IL-6 maximum, pg/mL & $957(1787) / 314$ & $545(1142) / 22$ & $933(1803) / 218$ & $1148(1884) / 74$ & 0.14 \\
\hline LDH maximum, U/L & $648(382) / 828$ & $566(339) / 162^{a}$ & $659(394) / 467$ & $686(379) / 199$ & $<0.001$ \\
\hline Leukocytes maximum, $10^{3} / \mathrm{mL}$ & $15.5(10.0) / 843$ & $13.1(7.7) / 173^{\mathrm{a}}$ & $16.2(10.5) / 467$ & $15.9(10.3) / 203$ & 0.001 \\
\hline Procalcitonin maximum, $\mathrm{ng} / \mathrm{mL}$ & $4.1(9.9) / 756$ & $3.4(9.5) / 143$ & $3.1(7.3) / 434$ & $7.1(14.2) / 179^{c}$ & $<0.001$ \\
\hline Platelets maximum, $1000 / \mathrm{mm}^{3}$ & $387(153) / 852$ & $390(152) / 176$ & $377(149) / 473$ & $405(161) / 203^{c}$ & 0.03 \\
\hline Bilirubin, maximum, mg/dL & $2.3(3.7) / 803$ & $1.8(2.2) / 152^{\mathrm{a}}$ & $2.3(3.4) / 461$ & $2.9(4.8) / 190$ & 0.001 \\
\hline AST maximum, $\mathrm{U} / \mathrm{L}$ & $198(372) / 845$ & $145(274) / 175^{\mathrm{a}}$ & $199(346) / 470$ & $240(483) / 200$ & $<0.001$ \\
\hline Creatinine maximum, mg/dL & $1.8(1.7) / 851$ & $1.8(1.8) / 175$ & $1.7(1.5) / 473$ & $2.2(1.9) / 203^{c}$ & 0.02 \\
\hline Urea maximum, mg/dL & $115(161) / 764$ & $117(316) / 157$ & $106(69) / 423$ & $135(106) / 184^{c}$ & $<0.001$ \\
\hline NTProBNP, maximum pg/mL & $1996(4815) / 261$ & $2573(6712) / 41$ & $1684(4693) / 152^{b}$ & $2344(3590) / 68$ & 0.04 \\
\hline Haematocrit minimum, \% & $38.2(6.0) / 834$ & $37.0(6.4) / 171$ & $38.8(5.8) / 464^{b}$ & $37.8(5.7) / 199$ & 0.003 \\
\hline Lactate maximum, mmol/L & $0.4(0.8) / 692$ & $0.3(0.5) / 142$ & $0.4(0.7) / 392$ & $0.4(1.2) / 158$ & 0.33 \\
\hline
\end{tabular}

Statistically significant results are in italics

${ }^{a}$ Never significantly different from early and delayed

${ }^{b}$ early significantly different from never and delayed

c delayed significantly different from never and early

SOFA: Sequential Organ Failure Assessment; CRP: C-reactive protein; LDH: lactate dehydrogenase; AST: aspartate Aminotransferase; NTProBNP: N-terminal probrain natriuretic peptide

consider this period long enough for an experienced clinician to assess patients' response to initial support and therapy and modify them accordingly. We have tried to solve the question placed by many clinicians: shall I start corticosteroids in this patient? Like an intention-to-treat analysis, patients receiving corticosteroids after $48 \mathrm{~h}$ of ICU admission were not excluded because the decision to start therapy in this group was probably guided by other uncontrolled factors.

Several recent publications support the early and selective use of corticosteroids in symptomatic patients infected with SARS-COV-2. The RECOVERY trial found a reduction in 28-day mortality in hospitalized COVID19 patients treated with dexamethasone if they required oxygen or MV [4]. The prospective meta-analysis of 7 
Table 3 Outcomes according to early corticosteroids use in the first $48 \mathrm{~h}$ of ICU admission, compared with never or delayed use

\begin{tabular}{|c|c|c|c|c|c|c|}
\hline & Whole cohort & Never & Early & p value & Delayed & $\mathrm{p}$ value \\
\hline $\begin{array}{l}\text { n (\%) } \\
\text { n IP-weighted (\%) }\end{array}$ & $\begin{array}{l}882 \\
455\end{array}$ & $\begin{array}{l}191(21.7 \%) \\
84(18.5 \%)\end{array}$ & $\begin{array}{l}485(55.0 \%) \\
259(57.0 \%)\end{array}$ & & $\begin{array}{l}206(23.4 \%) \\
112(24.6 \%)\end{array}$ & \\
\hline ICU mortality, cases/person-days & $308(34.9 \%) / 57,588$ & $70(36.6 \%) / 11,893$ & $147(30.3 \%) / 33,147$ & & $91(44.2 \%) / 12,547$ & \\
\hline ICU mortality (hazard ratio) & & 1 (Ref.) & $0.75(0.56-1.00)$ & 0.05 & $1.10(0.81-1.50)$ & 0.54 \\
\hline $\begin{array}{l}\text { ICU mortality (cases/person-days; hazard } \\
\text { ratio) IP-weighted }\end{array}$ & $116(25.5 \%) / 33,198$ & $\begin{array}{l}21(25 \%) / 5982 \\
1 \text { (Ref.) }\end{array}$ & $\begin{array}{l}52(20.1 \%) / 19,821 \\
0.55(0.31 .0 .95)\end{array}$ & 0.02 & $\begin{array}{l}43(38.4 \%) / 7394 \\
1.06(0.61-1.85)\end{array}$ & 0.84 \\
\hline 7-day mortality, cases/person-days & $72(8.2 \%) / 4229$ & $29(15.2 \%) / 864$ & $35(7.2 \%) / 2355$ & & $8(3.9 \%) / 1009$ & \\
\hline 7-day mortality (hazard ratio) & & 1 (Ref.) & $0.45(0.27-0.73)$ & 0.001 & $0.24(0.11-0.52)$ & $<0.001$ \\
\hline $\begin{array}{l}\text { 7-day mortality (cases/person-days; } \\
\text { hazard ratio) IP-weighted }\end{array}$ & $27(6.0 \%) / 2205$ & $\begin{array}{l}12(14.3 \%) / 377 \\
1 \text { (Ref.) }\end{array}$ & $\begin{array}{l}11(4.2 \%) / 1276 \\
0.23(0.10-0.55)\end{array}$ & $<0.001$ & $\begin{array}{l}4(3.6 \%) / 553 \\
0.20(0.06-0.62)\end{array}$ & 0.006 \\
\hline ICU length of stay, days & $17.8(14.2)$ & $14.2(13.0)$ & $16.4(12.5)$ & & $24.7(16.7)$ & \\
\hline ICU length of stay (mean difference) & & 0 (Ref.) & $2.2(-0.1$ to 4.5$)$ & 0.06 & $10.6(7.9-13.3)$ & $<0.001$ \\
\hline $\begin{array}{l}\text { ICU length of stay (days; mean differ- } \\
\text { ence) IP-weighted }\end{array}$ & $18.1(15.0)$ & $\begin{array}{l}13.0(12.7) \\
0 \text { (Ref.) }\end{array}$ & $\begin{array}{l}16.1(12.3) \\
2.6(-1.0 \text { to } 6.3)\end{array}$ & 0.16 & $\begin{array}{l}26.6(18.5) \\
13.0(8.2 .17 .9)\end{array}$ & $<0.001$ \\
\hline ICU length of stay among survivors, days & $18.0(14.7)$ & $15.0(13.0)$ & $16.6(13.1)$ & & $25.7(18.1)$ & \\
\hline $\begin{array}{l}\text { ICU length of stay among survivors } \\
\text { (mean difference) }\end{array}$ & & 0 (Ref.) & $1.6(-1.4$ to 4.6$)$ & 0.29 & $10.7(7.1-14.4)$ & $<0.001$ \\
\hline $\begin{array}{l}\text { ICU length of stay among survivors } \\
\text { (days; mean difference) IP-weighted }\end{array}$ & $17.7(15.0)$ & $\begin{array}{l}13.7(12.4) \\
0 \text { (Ref.) }\end{array}$ & $\begin{array}{l}15.9(12.6) \\
2.0(-1.7 \text { to } 5.7)\end{array}$ & 0.29 & $\begin{array}{l}26.7(19.7) \\
12.9(7.1-18.7)\end{array}$ & $<0.001$ \\
\hline Ventilatory-free days & $8.4(9.4)$ & $9.6(10.1)$ & $9.6(9.5)$ & & $4.7(7.3)$ & \\
\hline Ventilatory-free days (mean difference) & & 0 (Ref.) & $-0.00(-1.5$ to 1.5$)$ & 0.99 & $-4.9(-6.70$ to -3.09$)$ & $<0.001$ \\
\hline $\begin{array}{l}\text { Ventilatory-free days (days; mean differ- } \\
\text { ence) IP-weighted }\end{array}$ & $9.9(9.4)$ & $\begin{array}{l}12.3(9.9) \\
0 \text { (Ref.) }\end{array}$ & $\begin{array}{l}11.3(9.3) \\
0.23(-2.41 \text { to } 2.87)\end{array}$ & 0.86 & $\begin{array}{l}5.0(7.4) \\
-5.9(-8.7 \text { to }-3.5)\end{array}$ & $<0.001$ \\
\hline Medical complications, n (\%) & $860(97.5 \%)$ & $179(93.7 \%)$ & $477(98.3 \%)$ & & $204(99.0 \%)$ & \\
\hline Medical complications (odds ratio) & & 1 (Ref.) & $4.00(1.61-9.94)$ & 0.003 & $6.84(1.51-30.96)$ & 0.01 \\
\hline $\begin{array}{l}\text { Medical complications ( } \mathrm{n} \% \text {; odds ratio) } \\
\text { IP-weighted }\end{array}$ & $443(97.4 \%)$ & $\begin{array}{l}78(92.9 \%) \\
1 \text { (Ref.) }\end{array}$ & $\begin{array}{l}255(98.5 \%) \\
3.72(1.0-13.87)\end{array}$ & 0.05 & $\begin{array}{l}110(98.21 \%) \\
2.95(0.57-15.32)\end{array}$ & 0.20 \\
\hline Infectious complications, n (\%) & $509(57.7 \%)$ & $92(48.2 \%)$ & $273(56.3 \%)$ & & $144(69.9 \%)$ & \\
\hline Infectious complications (odds ratio) & & 1 (Ref.) & $1.39(0.99-1.94)$ & 0.05 & $2.50(1.66-3.77)$ & $<0.001$ \\
\hline $\begin{array}{l}\text { Infectious complications (n \%; odds } \\
\text { ratio) IP-weighted }\end{array}$ & $259(56.9 \%)$ & $\begin{array}{l}35(41.7 \%) \\
1 \text { (Ref.) }\end{array}$ & $\begin{array}{l}139(53.7 \%) \\
1.54(0.89-2.66)\end{array}$ & 0.12 & $\begin{array}{l}85(75.9 \%) \\
4.29(2.23-8.25)\end{array}$ & $<0.001$ \\
\hline
\end{tabular}

Statistically significant results are in italics

randomized trials performed by the WHO Rapid Evidence Appraisal for COVID-19 Therapies Working Group found that 28-day all-cause mortality in critically ill patients with COVID-19 was lower among those who received corticosteroids compared with those who received usual care or placebo (summary odds ratio, 0.66 ) [5]. Our study reinforces those results and sheds more light by providing new and more complete information. Of note, these findings are in contrast with previous reports on corticosteroid therapy in past outbreaks of other coronaviruses (SARS-CoV, MERS-CoV), or other viral pneumonia (Influenza, Respiratory Syncytial Virus) [12-16].

Our sensitivity analysis showed that early use of corticosteroids was not as effective in women, in those with lower risk of death -younger patients with good oxygenation and less inflammation-and neither in those with greater risk or severity -cancer, diabetics,
D-Dimer $>1500 \mathrm{ng} / \mathrm{mL}$, APACHE score $>14-$. These findings suggest that patient characteristics should be assessed before prescribing corticosteroids.

In clinical practice, corticosteroids are used in most critically ill patients [16]. The extensive use of corticosteroids in our cohort reflects the severity of our patients (almost 60\% directly admitted to ICU upon arrival to the hospital, $81.9 \%$ with invasive mechanical ventilation in the first $48 \mathrm{~h}$ after ICU admission, and with a mean $\mathrm{PaO} 2 / \mathrm{FiO} 2=150$ ). Since observational studies are prone to selection bias, we used inverse probability of treatment weighting to tackle this problem. Corticosteroid therapy is further entangled by other factors that merit discussion, including timing, type of corticosteroids, duration of treatment, and dosing.

Our average time to corticosteroids administration was 12 days after symptom onset (Additional file 2: Table S1), like the 13 days in the RECOVERY study for mechanically 

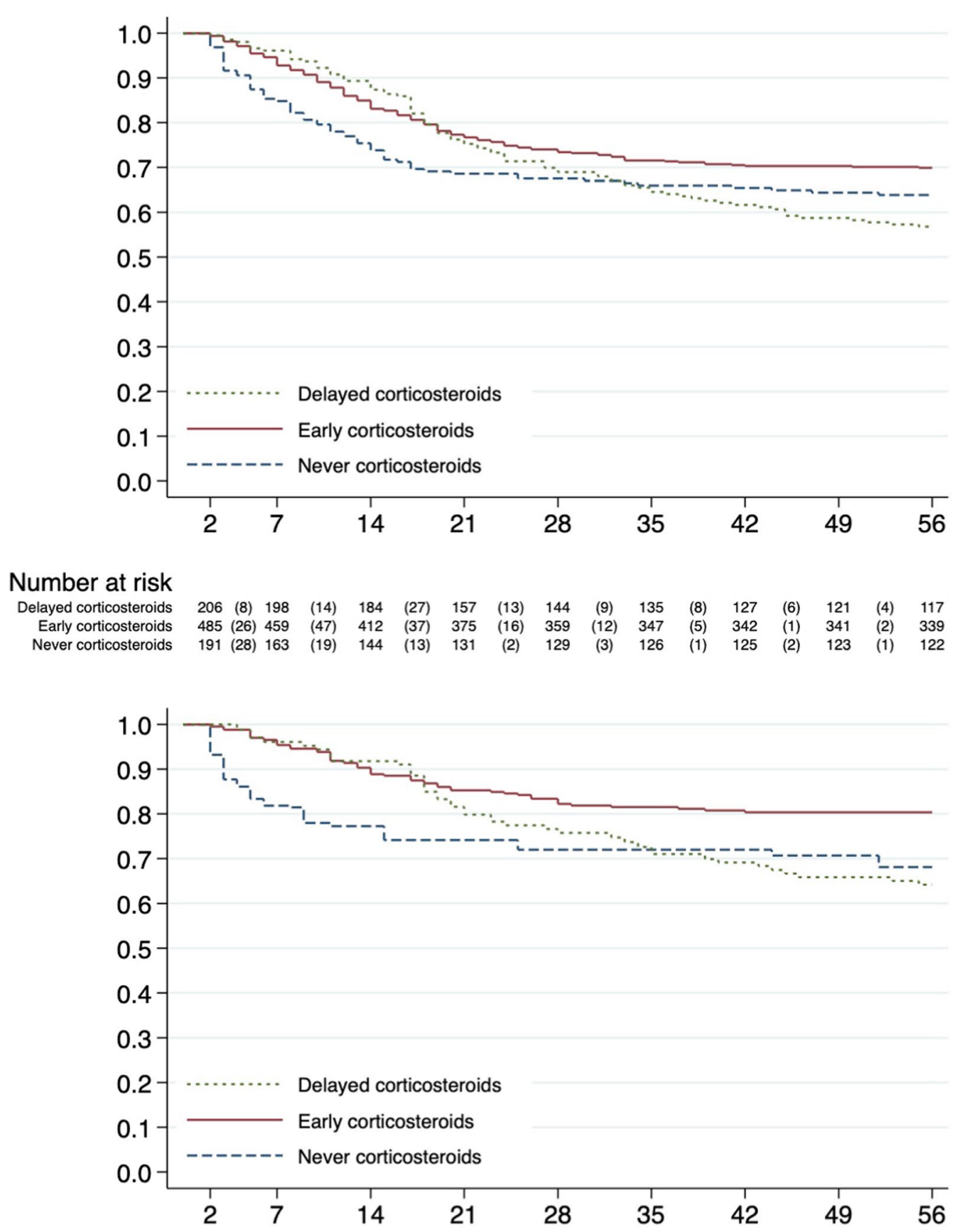

Number at risk

Early corticosteroids

Never corticosteroids

$\begin{array}{ccc}113 & 108 & 104 \\ 258 & 249 & 233 \\ 83 & 68 & 64\end{array}$

$\begin{array}{lr}104 & 92 \\ 64 & 220 \\ 64 & 62\end{array}$

$\begin{array}{cccccc}92 & 87 & 82 & 78 & 74 & 72 \\ 220 & 215 & 210 & 208 & 207 & 207 \\ 62 & 60 & 60 & 60 & 59 & 57\end{array}$

Fig. 2 Kaplan-Meier estimates of mortality according to the use of corticosteroids. Delayed $=$ after $48 \mathrm{~h}$ of ICU admission. The upper graphs are crude estimates. The lower graphs are inverse probability weighted (IPW) estimates $(\mathrm{N}=455)$

ventilated patients [4]. The decision to initiate corticosteroid therapy in our patients was guided when signs of hyperinflammation and severity of respiratory failure were evident. The delay or non-use of corticosteroids in $45 \%$ of our patients may reflect the controversy on their benefit/harm profile, WHO recommendations [1], 


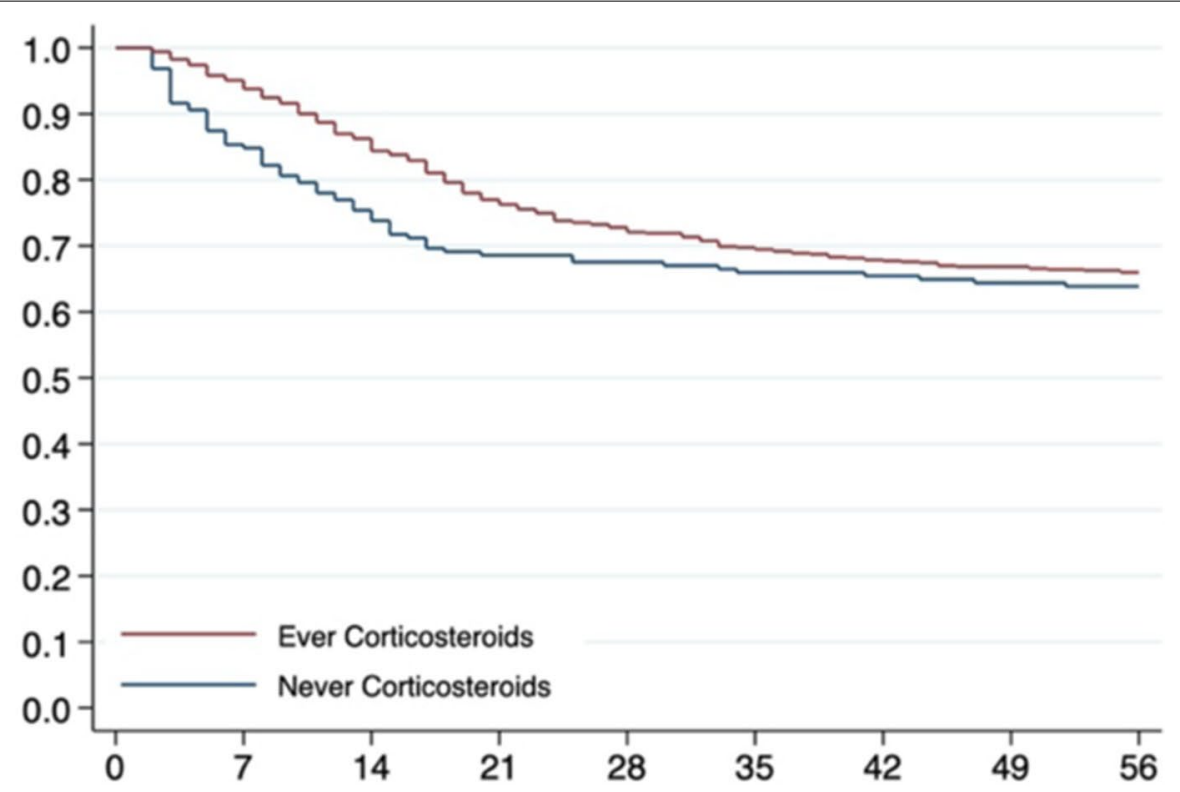

Number at risk

$\begin{array}{llllllllllllllllll}\text { Ever Corticosteroids } & 691 & (34) & 657 & (61) & 596 & (64) & 532 & (29) & 503 & (21) & 482 & \text { (13) } & 469 & \text { (7) } & 462 & (6) & 456\end{array}$ Never Corticostoroids $\begin{array}{lllllllllllllllll}691 & (34) & 657 & (61) & 596 & (64) & 532 & (29) & 503 & (21) & 482 & (13) & 469 & (7) & 462 & (6) & 456 \\ 191 & (28) & 163 & (19) & 144 & (13) & 131 & (2) & 129 & (3) & 126 & (1) & 125 & \text { (2) } & 123 & (1) & 122\end{array}$

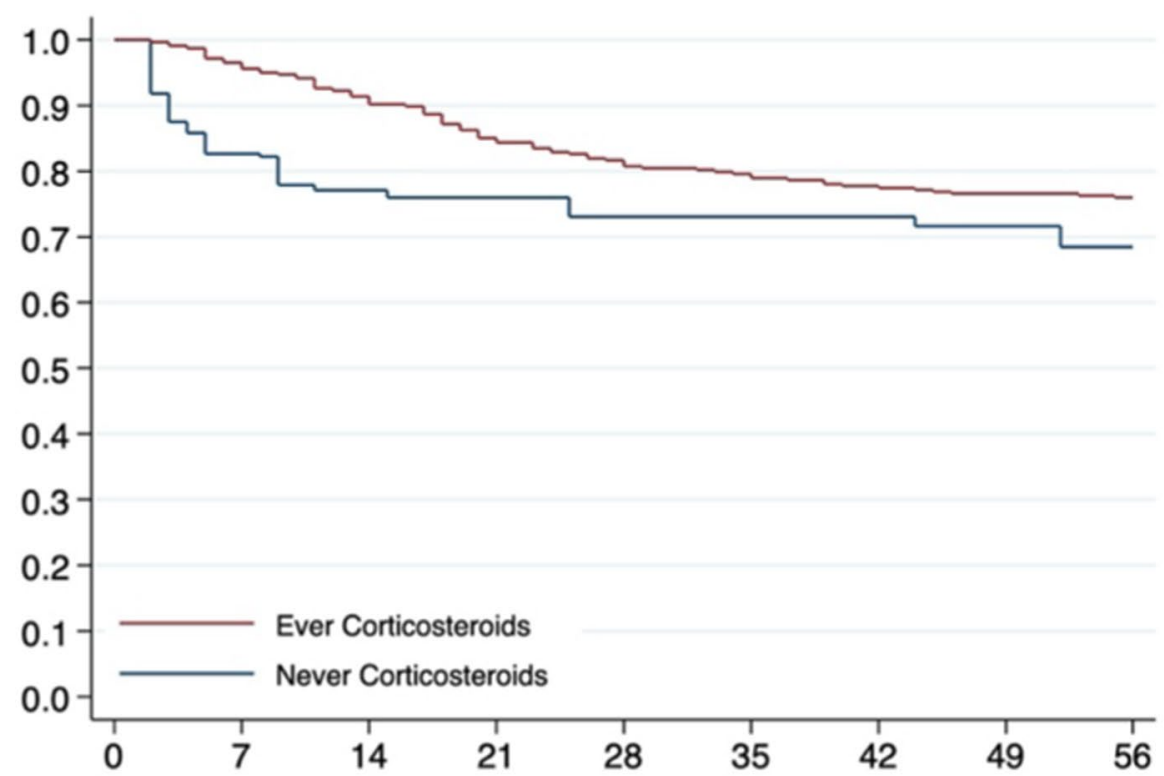

\section{Number at risk}

$\begin{array}{rccccccccc}\text { Ever Corticosteroids } & 322 & 311 & 294 & 274 & 263 & 256 & 250 & 246 & 245 \\ \text { Never Corticosteroids } & 70 & 58 & 54 & 53 & 51 & 51 & 51 & 50 & 48\end{array}$

Fig. 3 Kaplan-Meier estimates of mortality according to corticosteroids use during total hospital stay: ever (early + delayed) or never treatment. The upper graphs are the crude estimates. The lower graphs are IP-weighted estimates $(N=392)$ 
or significant changes in clinical evolution. Ideally, we should start corticosteroid therapy in the initial phases of the hyperinflammatory state. Early use in the absence of hyperinflammation could be harmful, especially in the initial stage of viral replication $[4,17,18]$. Similar to the RECOVERY trial, we have also found that corticosteroids were not associated with a reduction in mortality among those patients with symptoms duration under 7 days. One possible explanation is that corticosteroids may slow viral clearance in such an early phase. However, studies on viral clearance have yielded contradictory results [19-22]. Unfortunately, we did not collect time to viral clearance.

Interestingly, patients who never received corticosteroids had higher mortality than early treated patients, a difference that was not observed when we compare them with ever-treated patients, that includes early plus delayed treatment. Delayed corticosteroids, when advanced organ damage already exists, might be ineffective and even detrimental, as previously described in ARDS [23], with increased infectious complications and mortality, counteracting the positive effect of early treatment. The absence of benefit with too early use of corticosteroids (within 7 days of symptom onset), together with the beneficial effect of early use, and the worst results with delayed use, reveals a U-shaped timeoutcome relationship. Although we do not know the optimal time to start corticosteroids, probably patients with elevated inflammatory markers after seven days of symptoms, requiring oxygen or ventilatory support may benefit the most, whereas those who have not received corticosteroids in early phases, probably will not benefit afterwards.

To the best of our knowledge, recommendations about dose and duration of corticosteroid treatment are empiric. With large doses and long treatments, the potential for adverse effects increases, and the possible benefit is lost [24]. The Italian National Institute for Infectious Diseases recommends a 10-day regimen: 5-days full dose of methylprednisolone $1 \mathrm{mg} / \mathrm{Kg}$ daily or dexamethasone $20 \mathrm{mg}$ daily, and five days for tapering [25]. In general, most regimens for acute hyperinflammatory states recommend treatments shorter than two weeks $[25,26]$. Most common dose regimens range between $0.5-2 \mathrm{mg} /$ $\mathrm{kg} /$ day of methylprednisolone, equivalent to $0.1-0.4 \mathrm{mg} /$ $\mathrm{kg} /$ day of dexamethasone, defined in our study as moderate-to-high doses. The cut-off points were defined before the publication of the RECOVERY trial that used a fixed dexamethasone dose $(6 \mathrm{mg})$ lower than described in the literature for ARDS [10]. We observed a greater mortality reduction with a moderate-to-high dose regimen, similar to other studies $[6,9,27]$. Although our classification is artificial, it favours a higher dose of corticosteroids. As a result of the RECOVERY trial, the NIH and the WHO recommend a low fixed dose of daily dexamethasone [7, $8]$, and numerous studies with different corticosteroids and doses were prematurely stopped without completion $[5,9]$, but future comparative studies with higher doses are warranted $[6,10]$.

Also, the beneficial effects of early corticosteroids in our patients expand beyond an absolute reduction in allcause mortality, including a shorter ICU LOS, less organ dysfunction, and an increase in ventilator-free days.

This study has several strengths. First, this multicentre nationwide prospective data collection with over 1,000 patients from 36 ICUs provides a very detailed description of all gathered data from ICU admission to death or ICU discharge. Second, to the best of our knowledge, this is the first observational study that prospectively explores the association between different doses and timings of corticosteroid therapy in COVID-19 patients and ICU mortality. Third, we have used IPW to control for confounding with pre-specified demographic, comorbidities, and severity parameters. However, we acknowledge some limitations of our study. First, the observational nature of our study design, which may be subject to biases. Although we adjusted for likely confounders, some unmeasured confounding is still possible. Second, we cannot exclude missing data for some variables and potential for inaccuracies in the electronic health records due to the burden of care experienced by participating clinicians during the pandemic. However, due to the nature of our registry, we consider that selection bias was not favoured, and our analyses are valid. Third, although moderate-to-high doses of dexamethasone were most effective, no firm conclusions can be drawn on the drug or the dose, as our hypothesis and the definition of variables in the protocol preclude to do so, and doses predefined as low are not completely equivalent and could be controversial. Finally, at the time of the analysis, 149 (13.5\%) patients did not have a definitive outcome regarding status at ICU discharge and were not included in the main analysis, although included in the sensitivity analyses.

\section{Conclusions}

In conclusion, in critically ill COVID-19 patients with acute respiratory failure, the use of corticosteroids within the first $48 \mathrm{~h}$ of ICU admission was associated with a marked reduction in ICU mortality and ICU LOS. We also found a clear relationship between exposure and a beneficial effect on organ dysfunction. Further research is needed to characterize the optimal drug, onset, dose, and duration of corticosteroids therapy in this patient population. 


\section{Supplementary information}

Supplementary information accompanies this paper at https://doi. org/10.1186/s13054-020-03422-3.

Additional file 1. Methods, Tables S3-6, Figures S2-5 and References.

Additional file 2. Table S1: Use of corticosteroids and combination of treatments.

Additional file 3. Table S2: Sensitivity analyses: ICU mortality comparing early versus non-earlyuse of corticosteroids.

Additional file 4. Figure S1: Restricted cubic spline to plot the odds ratio ( $95 \%$ confidenceinterval) of mortality according to the onset day of corticosteroids treatment.

\section{Abbreviations}

APACHE: Acute Physiology and Chronic Health disease Classification System; ARDS: Acute respiratory distress syndrome; Cl: Confidence interval; COVID-19: Coronavirus disease 2019; CRP: C-reactive protein; HR: Hazard ratio; ICU: Intensive Care Unit; IPW: Inverse probability of treatment weighting; IQR: Interquartile range; LOS: Length of stay; MV: Mechanical ventilation; $\mathrm{NIH}$ : US National Institutes of Health; RRT: Renal replacement therapy; SARS-CoV-2: Severe acute respiratory syndrome coronavirus 2; SD: Standard deviation; SOFA: Sequential Organ Failure Assessment; WHO: World Health Organization.

\section{Acknowledgements}

We thank all patients and their families involved in the study. We would like the names of the individual members of the COVID-19 Spanish ICU Network Group to be searchable through their individual PubMed records.

Investigators of the COVID-19 Spanish ICU Network are: Hospital Clínic de Barcelona, Department of Anesthesiology and Critical Care: Marina Vendrell, Gerard Sánchez-Etayo, Amalia Alcón, Isabel Belda, Mercé Agustí, Albert Carramiñana, Isabel Gracia, Miriam Panzeri, Irene León, Jaume Balust, Ricard Navarro, María José Arguís, María José Carretero, Cristina Ibáñez, Juan Perdomo, Antonio López, Manuel López-Baamonde, Tomás Cuñat, Marta Ubré, Antonio Ojeda, Andrea Calvo, Eva Rivas, Paola Hurtado, Roger Pujol, Nuria Martín, Javier Tercero, Pepe Sanahuja, Marta Magaldi, Miquel Coca, Elena del Rio, Julia Martínez-Ocon, Paula Masgoret, Monserrat Tio, Angel Caballero, Raquel Risco, Raquel Bergé, Lidia Gómez, Nicolás de Riva, Ana Ruiz, Beatriz, Tena, Sebastián Jaramillo, José María Balibrea, Francisco Borja de Lacy, Ana Otero, Ainitze Ibarzabal, Raquel Bravo, Anna Carreras, Daniel Martín-Barreda, Alfonso Jesús Alias, Mariano Balaguer, Jorge Aliaga, Alex Almuedo, Joan Ramón Alonso, Rut Andrea, Gerard Sergi Angelès, Marilyn Arias, Fátima Aziz, Joan Ramon Badía, Enric Barbeta, Toni Torres, Guillem Batiste, Pau Benet, Xavi Borrat, María Borrell, Ernest Bragulat, Inmaculada Carmona, Manuel Castellà, Pedro Castro, Joan Ceravalls, Oscar Comino, Claudia Cucciniello, Clàudia De Deray, Oriol De Diego, Paula De la Matta, Marta Farrero, Javier Fernández, Sara Fernández, Anna Fernández, Miquel Ferrer, Ana Fervienza, María Tallo Forga, Daniel Forné, Clàudia Galán, Andrea Gómez, Eduard Guasch, María HernándezTejero, Adriana Jacas, Beltrán Jiménez, Pere Leyes, Teresa López, José Antonio Martínez, Graciela Martínez-Pallí, Jordi Mercadal, Guido Muñoz, José Muñoz, Ricard Navarro, Josep María Nicolás, José Tomás Ortiz, Anna Peiró, Manuel Pérez, Esteban Poch, Margarida Pujol, Eduard Quintana, Bartomeu Ramis, Enric Reverter, Irene Rovira, Pablo Ruiz, Elena Sandoval, Stefan Schneider, Oriol Sibila, Carla Solé, Alex Soriano, Dolors Soy, M. Suárez, Adrián Téllez, Néstor David Toapanta, Antoni Torres, Xavier Urra. Hospital Universitario Río Hortega: César Aldecoa, Alicia Bordell, Silvia Martín, Judith Andrés. Hospital Universitario Cruces: Alberto Martínez Ruiz, Gonzalo Tamayo Medel, Iñaki Bilbao Villasante, Fernando Iturri Clavero, Covadonga Peralta Álvarez, Julia T. Herrera Díez, Andrea García Trancho, Iñaki Sainz Mandiola, Carmen Ruano Suarez, Angela Ruiz Bocos, Eneritz Urrutia Izagirre, Pablo Ortiz de Urbina Fernández, Naiara Apodaka López, Leire Prieto Molano, Eunate Ganuza Martínez, Iratxe Vallinas Hidalgo, Karmele de Orte Sancho, Celia González Paniagua, Gemma Ortiz Labrador, Mireia Pérez Larrañaga, Marta López Miguelez, Estíbaliz Bárcena Andrés, Erik Urutxurtu Laureano, Maria Jesús Maroño Boedo, Blanca Escontrela Rodríguez, Aitziber Ereñozaga Camiruaga, Deiene Lasuen Aguirre, Ainhoa Zabal Maeztu, Ane Guereca Gala, Iker Castelo Korro, Andrés Álvarez Campo, Alejandro Carcelen Viana, Alejandro Alberdi Enríquez, Xabier Ormazábal Rementeria, Alberto Sánchez Campos, Rosa Gutiérrez Rico, Pablo Barbier
Damborenea, Marta Guerenabarrena Momeñe, Borja Cuesta Ruiz, Alejandro López Rico, Ana Rojo Polo, Covadonga García Grijelmo, Mikel Celorrio Reta, Eneko Martín Arroyo, Leire Artaza Aparicio, Iñaki Ituarte Aspiazu, Ane Igeregi Basabe, Itxaso Merino Julian, Isabel Diaz Rico, Maria Paz Martínez. Hospital del Mar: Ramón Adalia Bartolomé, Luigi Zattera, Irina Adalid Hernandez, Leire Larrañaga Altuna, Aina Serrallonga Castells, Adriana Vílchez Garcia, María Núñez, Lorena Román, Isabel Ramos Delgado, Adela Benítez-Cano Martínez, Mireia Chanzá Albert, Juan Carlos Álvarez García, Luis Aguilera Cuchillo, Sandra Beltrán de Heredia, Jesús Carazo Cordobés, Carlos Alberto García Bernedo, Fernando Escolano Villén. Hospital General Universitario de Ciudad Real: Francisco Javier Redondo Calvo, Rubén Villazala González, Victor Baladron González, Patricia Faba, Omar Montenegro, Natalia Bejarano Ramírez. Complejo Asistencial Universitario León: Sergio Marcos Contreras, Alejandro Garcia Rodríguez, Saleta Rey Vázquez, Cristina Garcia Pérez, Eva Higuera Miguelez, Irene Pérez Blanco, David García Rivera. Hospital Urduliz: Ane Martín de la Fuente, Marta Pardo, Vanessa Rodriguez, Unai Bengoetxea. Hospital Universitario de la Princesa: Fernando Ramasco, Sheila Olga Santidrián Bernal, Alvar Santa Cruz Hernando, Antonio Planas Roca, Carlos Figueroa Yusta, Esther García Villabona, Carmen Vallejo Lantero, Eva Patiño Rodriguez, Alvaro Esquivel Toledo, David Arribas Méndez, Mar Orts Rodriguez, Rosa Méndez Hernández, Jesús Nieves Alonso, Inés Imaz Artazcoz, Sonia Expósito Carazo, Carlos Román Guerrero, Elena Rojo Rodríguez, Ricardo Moreno González, Julia Hernando Santos, Jara Torrente Pérez, Esperanza Mata Mena, Manuel José Muñoz Martínez, Enrique Alday Muñoz, Patricia Martin Serrano, Laura Cotter Muñoz, Amadea Mjertan, Diego Gutierrez Martínez, Carmen Rodríguez García, Olaya Alonso Viejo, Juan Alvarez Pereira, Ana Carmona Bonet, Diana Parrado López, Eva de Dios Tomas, Rafael Martín Celemin, María Luisa Meilan Paz, Luis Quecedo Gutiérrez, Noemí Diaz Velasco, Gabriel Martin Hernández, Francisco Garcia del Corral, Gloria Hernandez Arias, David Rodriguez Cuesta, Ana Gómez Rice, Encarna Mateos Sevillano, Natalia Olmos Molpeceres. Hospital Povisa: Beatriz Domínguez, Ana Vázquez Lima. Hospital Ramón y Cajal: Ángel Candela, Ismael A Acevedo Bambaren, Maria Isabel Albala Blanco, Paloma Alonso Montoiro, Fernando Álvarez Utrera, Juan Avellanosa Esteruelas, Amal Azzam López, Alberto José Balvis Balvis, Tommaso Bardi, María Beltrán Martín, Jacobo Benatar Haserfaty, Alberto Berruezo Camacho, Laura Betolaza Weimer, María del Mar Carbonell Soto, Cristina Carrasco Seral, Cristina Cerro Zaballos, Elizabeth Claros Llamas, Pilar Coleta Orduna, Ingrid P. Cortes Forero, Pascual Agustín Crespo Aliseda, María Angélica de Pablo Pajares, Yolanda Díez Remesal, Trinidad Dorado Díaz, Noemí Echevarría Blasco, María Elena Elías Martín, Javier Felices Triviño, Natalia Fernández López, Cristina Fernández Martín, Natalia Ferreiro Pozuelo, Luis Gajate Martín, Clara Gallego Santos, Diego Gil Mayo, María Gómez Rojo, Claudia González Cibrián, Elena Herrera López, Borja Hinojal Olmedillo, Berta Iglesias Gallego, Sassan Khonsari, María Nuria Mane Ruiz, María Manzanero Arroyo, Ana María Mariscal Ortega, Sara Martín Burcio, María del Carmen Martín González, Ascensión Martín Grande, Jose Juan Martín López, Cecilia Martín Rabes, Marcos Martínez Borja, Nilda Martínez Castro, Adolfo Martínez Pérez, Snejana Matcan, Cristina Medrano Viñas, Lisset Miguel Herrera, Adrián Mira Betancur, María Montiel Carbajo, Javier Moya Moradas, Lorena Muñoz Pérez, Mónica Nuñez Murias, Eva Ordiales González, Óscar Ordoñez Recio, Miguel Ángel Palomero Rodriguez, Diego Parise Roux, Lucia Pereira Torres, David Pestaña Lagunas, Juana María Pinto Corraliza, Marian Prieto Rodrigo, Inmaculada Rodriguez Diaz-Regaño, David Rodriguez Esteban, Víctor Rojas Pernia, Álvaro Ruigómez Saiz, Bárbara Saavedra Villarino, Noemí Samaranch Palero, Gloria Santos Pérez, Jaume Serna Pérez, Ana Belén Serrano Romero, Jesús Tercero López, Carlos Tiscar García, Marta de la Torre Concostrina, Eva María Ureta Mesa, Eva Velasco Olarte, Judith Villahoz Martínez, Raúl Villalaba Palacios, Gema Villanueva García, Cristina Vogel de Medeiros. Hospital Universitario Severo Ochoa: Soraya Gholamian Ovejero, Marta Vicente Orgaz, Patricia Lloreda Herradon, Cristina Crespo Gómez. Hospital Universitario de Gran Canaria Dr. Negrín: Tatiana Sarmiento-Trujillo. Hospital de Terrassa: Noemí García Medina, María Martínez García, Carles Espinós Ramírez, Nabil Mouhaffel Rivero, Jose Antonio Bernia Gil. Hospital Central de la Cruz Roja San José y Santa Adela: Sonsoles Martín. Hospital de la Santa Creu i Sant Pau: María Victoria Moral, Josefina Galán, Pilar Paniagua, Sergio Pérez, Albert Bainac, Ana Arias, Elsa Ramil, Jorge Escudero. Clínica Universidad de Navarra: Departamento de Anestesiología y Cuidados Intensivos: Pablo Monedero, Carmen Cara, Andrea Lara, Elena Mendez Martínez, Jorge Mendoza, Iñigo Rubio Baines, Carmen Sala Trull, Pablo Montero López. Medicina Preventiva y Salud Pública: Alfredo Gea, Alejandro Montero. Hospital Universitario Dr. Peset Aleixandre: Rocío Armero Ibañez, Juan Vicente Llau Pitarch, Fernando Rauer Alcóver, Cristina Álvarez Herreros, 
Cyntia Sánchez Martín, Lucía López Ocáriz Olmos, Marta Navas Moruno. Complejo Hospitalario Universitario de Cáceres: Fernando García Montoto. MF. Mirón Rodriguez, Laura Fuentes Coco, Cristina Hernández Gamito, Antonio Barba Orejudo, Luis Gerardo Smith Vielma, Yasmina González Marín, Francisco de Borja Amador Penco, Marta Donoso Domínguez, Silvia Esquivel Ramírez. Hospital Clínico Universitario de Valencia: José Antonio Carbonell, Berta Monleón López, Sara Martínez-Castro, Gerardo Aguilar. Hospital Universitario a Coruña: María Gestal, Pablo Casas, Angel Outeiro Rosato, Andrea Naveiro Pan, María Alonso Portela, Adrián García Romar, Eva Mosquera Rodríguez, Diego Ruanova Seijo, Pablo Rama Maceiras. Complexo Universitario de Ferrol: Francisco Castro-Ceoane, Esther Moreno López. Hospital Clínico Universitario Lozano Blesa: Sergio Gil, Julia Guillén Antón, Patricia García-Consuegra Tirado, Aurora Callau Calvo, Laura Forés Lisbona, María Carbonell Romero, Belén Albericio Gil, Laura Pradal Jarne, María Soria Lozano, Diego Loscos López, Andrea Patiño Abarca. Universal Doctors: Jordi Serrano. UBIKARE: Javier Pérez-Asenjo, Ángel Díez-Domínguez, Ion Zubizarreta, Jon Ramos, losu Fernández. Hospital Universitario La Paz: Emilio Maseda, Alejandro Suárez de la Rica, Javier Veganzones, Itziar Insausti, Javier Sagra, Sofía Díaz Carrasco, Ana Montero Feijoo, Julio Yagüe. Hospital Universitario Gregorio Marañon: Ignacio Garutti, Javier Hortal, Patricia Piñeiro, Matilde Piñeiro, Matilde Zaballos, Jamil cedeño, Pablo García-Olivares, Alberto Garriido, Jose Eugenioi Guerrero. Hospital San Joan Despí Moises Broggi:Eva Bassas Parga, Carmen Deiros Garcia, Elisenda Pujol Rosa, Ana Tejedor Navarro, Roser Font Gabernet, Maria José Bernat, Meritxell Serra Valls, Cristina Cobaleda Garcia-Bernalt, Jesus Fernanz Anton, Adriana Aponte Sierra, Lucia Gil Gomez, Olaia Guenaga Vaqueiro, Susana Hernandez Marin, Laura Pardo Pinzon, Sira Garcia Aranda, Carlos Briones Orejuela, Edgar Cortes Sánchez, Alejandro Romero Fernández, Esther Fernández SanJosé, Patricia Iglesias Garsabal, Guillermo Isidro Lopez, Ana Vicol, Sara Espejo Malagon, María Sanabra Loewe, Laura Grau Torradeflo, Lourdes Blanco Alcaide, Gloria Buenaventura Sanclemente, Pere Serra Pujol, Gustavo Cuadros Mendoza, Miroslawa Konarska, Fedra Bachs Almenara, Agnieszka Golska, Aleix Carmona Blesa, Arantxa Mas Serra. Hospital Universitario Infanta Leonor: Javier Ripolles Melchor, Ana Nieto Moreno, Káteri Chao Novo, Sandra Gadín López, Elena Nieto Moreno, Bérénice Gutiérrez Tonal, Elena Lucena de Pablo, Barbara Algar Yañez, Beatriz Vázquez Rivero, Beatriz Nozal Mateo, Marina de Retes, Norma Aracil Escoda, Cristina Gallardo Mayo, Rosa Sanz González, Alicia Ruiz Escobar, Maria Laura Pelegrina López, Marina Valenzuela Peña, David Stolle Dueñas, Ane Abad Motos, Alfredo Abad-Gurumeta, Ana Tirado Errazquin, Elena Sáez Ruiz, Nerea Gómez Pérez, Francisco de Borja Bau González. Hospital sanitas CIMA: Cesar Morcillo Serra, Jessica Souto Higueras. Hospital Universitario y Politécnico La Fé: Rosario Vicente, Raquel Ferrandis, Silvia Polo Martín, Azucena Pajares Moncho, Ignacio Moreno Puigdollers, Juan Pérez Artacho Cortés, Ana Moret Calvo, Ana Pi Peña, María Catalán Fernández. Complexo hospitalario Universitario de Pontevedra: Marina Varela, Pilar Díaz Parada, Raquel Rey Carlín, Sarra Barreiro Aragunde. Hospital Arnau de Vilanova: María Isabel Forés Chiva. Hospital General de Alicante: A. Javier Agulló. Hospital Universitario Infanta Sofía: Antonio Pérez Ferrer. Hospital Universitario San Juan de Alicante: María Galiana. Hospital Nuestra Señora de Meritxell SAAS: Antoni Margarit, Válerie Mourre del Rio, Eva Heras Muxella, Anna Vidal.

\section{Authors' contributions}

PM, PC, ACT, MH, CF, and the COVID-19 Spanish ICU Network collected the clinical data. AG, EA, and CF performed the initial data analysis and interpretation. PM and PC summarized all data. ACT, AG, CF, PM, and JV drafted the manuscript. JV, MH, and PC contributed to data interpretation and revised the final manuscript. All authors contributed to critical revision of the manuscript for intellectual content, and all are responsible for the content of this paper. All authors read and approved the final manuscript.

\section{Funding}

None.

\section{Availability of data and materials}

After publication, data will be made available to other investigators on reasonable requests to the corresponding author. A proposal with a detailed description of study objectives and statistical analysis plan will be needed for evaluation of the reasonability of requests. Additional materials might also be required during the process of evaluation. Deidentified participant data will be provided after approval from the corresponding author.

\section{Ethics approval and consent to participate}

Approval was obtained from the referral Ethics Committee of Euskadi, Spain (by Nagore García Goitiandia. Secretaria del CElm de Euskadi. Código interno: PI2020069. Versión del Protocolo: VERSIÓN 5-5 de mayo-2020). The procedures used in this study adhere to the tenets of the Declaration of Helsinki.

\section{Consent for publication}

Not applicable.

\section{Competing interests}

We declare no competing interests.

\section{Author details}

${ }^{1}$ Department of Anaesthesiology and Intensive Care, Clínica Universidad de Navarra, Pio XII, 36, 31008 Pamplona, Spain. ${ }^{2}$ Department of Preventive Medicine and Public Health, Medical School, University of Navarra, Pamplona, Spain. ${ }^{3}$ Medical Intensive Care Unit, Hospital Clínic, Institut D'investigació August Pi i Sunyer (IDIBAPS), University of Barcelona, Barcelona, Spain. ${ }^{4}$ Department of Anesthesiology and Critical Care, Hospital del Ramón y Cajal, Madrid, Spain. ${ }^{5}$ Department of Anesthesiology and Critical Care, Hospital de Cruces, Barakaldo, Vizcaya, Spain. ${ }^{6}$ Ubikare Technology, Vizcaya, Spain. ${ }^{7}$ CIBER de Enfermedades Respiratorias, Instituto de Salud Carlos III, Madrid, Spain.

${ }^{8}$ Li Ka Shing Knowledge Institute, St Michael's Hospital, Toronto, ON, Canada.

${ }_{9}^{9}$ Multidisciplinary Organ Dysfunction Evaluation Research Network, Research Unit, Hospital Universitario Dr. Negrín, Las Palmas de Gran Canaria, Spain.

${ }^{10}$ Department of Anesthesiology and Critical Care, Hospital Clínic, Institut D'investigació August Pi i Sunyer, Barcelona, Spain.

Received: 7 September 2020 Accepted: 2 December 2020 Published online: 04 January 2021

\section{References}

1. WHO. Clinical management of COVID-19. https://www.who.int/publicatio ns/i/item/clinical-management-of-covid-19. Accessed 8 November 2020.

2. Felsenstein S, Herbert JA, McNamara PS, Hedrich CM. COVID-19: immunology and treatment options. Clin Immunol. 2020;215:108448.

3. Hodgens A, Sharman T. Corticosteroids. In: StatPearls. Treasure Island (FL): StatPearls Publishing; May 14, 2020.

4. RECOVERY Collaborative Group, Horby P, Lim WS, et al. Dexamethasone in Hospitalized Patients with Covid-19-preliminary report. N Engl J Med. 2020 Jul 17:NEJMoa2021436.

5. The WHO Rapid Evidence Appraisal for COVID-19 Therapies (REACT) Working Group. Association Between Administration of Systemic Corticosteroids and Mortality Among Critically III Patients With COVID-19: A Meta-analysis. JAMA. 2020;324(13):1330-1341.

6. Tomazini BM, Maia IS, Cavalcanti AB, et al. Effect of dexamethasone on days alive and ventilator-free in patients with moderate or severe acute respiratory distress syndrome and COVID-19: the CODEX randomized clinical trial. JAMA. 2020:324(13):1307-16.

7. Centers for Disease Control Prevention. Clinical Care Guidance for Healthcare Professionals about Coronavirus (COVID-19). https://www.cdc.gov/ coronavirus/2019-ncov/hcp/clinical-care.html. Accessed 8 November 2020.

8. WHO. Corticosteroids for COVID-19. https://www.who.int/publications/i/ item/WHO-2019-nCoV-Corticosteroids-2020.1. Accessed 8 November 2020.

9. De Backer D, Azoulay E, Vincent J. Corticosteroids in severe COVID-19: a critical view of the evidence. Crit Care. 2020;24:627-9.

10. Villar J, Ferrando C, Martínez D, et al. Dexamethasone treatment for the acute respiratory distress syndrome: a multicentre, randomised controlled trial. Lancet Respir Med. 2020;8(3):267-76.

11. Hernán MA, Hernández-Díaz S, Robins JM. A structural approach to selection bias. Epidemiology. 2004;15(5):615-25.

12. Li H, Chen C, Hu F, et al. Impact of corticosteroid therapy on outcomes of persons with SARS-CoV-2, SARS-CoV, or MERS-CoV infection: a systematic review and meta-analysis. Leukemia. 2020;34(6):1503-11.

13. Arabi YM, Mandourah Y, Al-Hameed F, et al. Corticosteroid therapy for critically ill patients with middle east respiratory syndrome. Am J Respir Crit Care Med. 2018;197(6):757-67. 
14. Guan WJ, Ni ZY, Hu Y, et al. Clinical characteristics of coronavirus disease 2019 in China. N Engl J Med. 2020;382(18):1708-20.

15. Russell CD, Millar JE, Baillie JK. Clinical evidence does not support corticosteroid treatment for 2019-nCoV lung injury. Lancet. 2020;395(10223):473-5.

16. Alhazzani W, Møller MH, Arabi YM, et al. Surviving Sepsis Campaign: guidelines on the management of critically ill adults with Coronavirus Disease 2019 (COVID-19). Intensive Care Med. 2020;46(5):854-87.

17. Johnson RM, Vinetz JM. Dexamethasone in the management of covid-19. BMJ. 2020;370:m2648.

18. Tang C, Wang Y, Lv H, Guan Z, Gu J. Caution against corticosteroid-based COVID-19 treatment. Lancet. 2020:395(10239):1759-60.

19. Fang X, Mei Q, Yang T, et al. Low-dose corticosteroid therapy does not delay viral clearance in patients with COVID-19. J Infect. 2020;81(1):147-78.

20. Jung J, Oh DK, Ahn JH, et al. Re: Low-dose corticosteroid therapy does not delay viral clearance in patients with COVID-19. J Infect. 2020;81(2):e79-81.

21. Ling $Y, X u$ SB, Lin $Y X$, et al. Persistence and clearance of viral RNA in 2019 novel coronavirus disease rehabilitation patients. Chin Med J (Engl). 2020;133(9):1039-43.

22. Zha L, Li S, Pan L, et al. Corticosteroid treatment of patients with coronavirus disease 2019 (COVID-19). Med J Aust. 2020;212(9):416-20.
23. Steinberg KP, Hudson LD, Goodman RB, et al. Efficacy and safety of corticosteroids for persistent acute respiratory distress syndrome. $\mathrm{N}$ Engl J Med. 2006;354(16):1671-84.

24. Theoharides TC, Conti P. Dexamethasone for COVID-19? Not so fast. J Biol Regul Homeost Agents. 2020;34:3.

25. Nicastri E, Petrosillo N, Ascoli Bartoli T, et al. National institute for the infectious diseases "L. Spallanzani", IRCCS recommendations for COVID-19 clinical management. Infect Dis Rep. 2020;12(1):8543.

26. Xu X, Ong YK, Wang Y. Role of adjunctive treatment strategies in COVID19 and a review of international and national clinical guidelines. Mil Med Res. 2020;7(1):22

27. Meduri GU, Siemieniuk RAC, Ness RA, Seyler SJ. Prolonged low-dose methylprednisolone treatment is highly effective in reducing duration of mechanical ventilation and mortality in patients with ARDS. J Intensive Care. 2018;6:53.

\section{Publisher's Note}

Springer Nature remains neutral with regard to jurisdictional claims in published maps and institutional affiliations.
Ready to submit your research? Choose BMC and benefit from:

- fast, convenient online submission

- thorough peer review by experienced researchers in your field

- rapid publication on acceptance

- support for research data, including large and complex data types

- gold Open Access which fosters wider collaboration and increased citations

- maximum visibility for your research: over $100 \mathrm{M}$ website views per year

At BMC, research is always in progress.

Learn more biomedcentral.com/submissions 